\title{
Results from the commissioning of the ATLAS Pixel Detector with cosmic ray data
}

\author{
I. Ibragimov ${ }^{*, a}$, on behalf of the ATLAS Collaboration \\ ${ }^{a}$ Fachbereich Physik, Universität Siegen, D-57068, Siegen, Germany
}

\begin{abstract}
The ATLAS Pixel Detector is one of the largest silicon pixel hybrid detectors in the world. It has a total active area of $1.7 \mathrm{~m}^{2}$ of silicon read out every $25 \mathrm{~ns}$ by approximately 80 million electronic channels. It is the innermost tracking detector of the ATLAS experiment at the Large Hadron Collider (LHC) at CERN, designed to measure particle tracks and decay vertices with a very high precision and efficiency. Since August 2008, after more than 10 years of development and construction, the whole detector has been operated together. After tuning, calibration and timing-in the detector has demonstrated excellent noise occupancy of $10^{-10}$ and a tracking hit efficiency greater than $99.7 \%$. The paper will describe the detector performance and discuss the studies performed with cosmic ray data, such as alignment and the Lorentz angle measurement.
\end{abstract}

Key words: Silicon pixel detectors, Commissioning, Alignment, Lorentz angle

\section{Introduction}

The ATLAS Pixel Detector $[1,2]$ has been designed to detect particle tracks and decay vertices near the collision point. The resolution of the detector is expected to be $10 \mu \mathrm{m}$ in the $R \phi$ plane (perpendicular to the beam axis) and $110 \mu \mathrm{m}$ in $z$ direction (parallel to the beam axis). This requires high granularity combined with low mass of the detector material. At the full LHC luminosity of $10^{34}$ $\mathrm{cm}^{-2} \mathrm{~s}^{-1}$ proton-proton collisions are expected every $25 \mathrm{~ns}$, which requires fast hit identification. The trigger latency of $3.2 \mu \mathrm{s}$ [3] requires local buffering of hits in the front-end readout electronics. The detector has been designed and constructed to tolerate the expected radiation dose of 500 kGy over 10 years lifetime, while providing a hit detection efficiency greater than $97 \%$ over that period and beyond. In order to fulfill all these requirements the silicon pixel hybrid technology has been chosen for the Pixel Detector design.

This paper presents first measurements with the detector after its installation in the ATLAS cavern. The results of the extensive calibration program performed in-situ provide a baseline characterization of the detector prior to the first radiation effects. Combined ATLAS data-taking periods with cosmic ray triggers have been extensively used as an excellent opportunity to study the detectors characteristics, to accumulate running experience and to perform the first system debugging studies.

A brief overview of the Pixel Detector is given in Section 2, followed by a summary of the commissioning ac-

\footnotetext{
${ }^{*}$ Corresponding author.

Email address: ibragimov@hep.physik.uni-siegen.de (I. Ibragimov)

Preprint submitted to Elsevier
}

tivities in Section 3. In Section 4 results of the analyses performed with the cosmic ray data are presented.

\section{Pixel Detector layout}

The Pixel Detector is built with 1744 identical modules, grouped into three cylindrical barrel layers around the beam axis at radii 5,9 and $12 \mathrm{~cm}$ and two endcaps, each consisting of three disks. This geometry provides three space-points for tracking in the pseudorapidity range $|\eta|<2.5$. Each Pixel module consists of the $250 \mu \mathrm{m}$ thick $n^{+}$-on- $n$ silicon pixel sensor with a $p^{+}$backplane. The total active area of the sensor is $6.08 \mathrm{~cm} \times 1.64 \mathrm{~cm}$ and the nominal pixel size is $400 \mu \mathrm{m} \times 50 \mu \mathrm{m}$.

The sensor is read out by 16 Front-End (FE) chips, which are bump-bonded to the sensor, with in total 46080 channels. Each channel has individually adjustable preamplifier and discriminator circuits. For each signal above the discriminator threshold, the timestamp of its leading edge and the measured charge as Time-over-Threshold (ToT) ${ }^{1}$ are stored. The timestamp is derived from the module replica of the $40 \mathrm{MHz}$ LHC clock. For the threshold tuning each pixel has a 7-bit DAC. The back side of the sensor is glued to the flexible printed circuit board with high density interconnections. This board is wire-bonded to the FE chips and houses an NTC thermistor for temperature measurement and a Module Controller Chip (MCC). Upon receiving the trigger command, the MCC can read out data from the FE's for up to 16 consecutive clock cycles and build a local event out of the received FE hits for each cycle. In this way a readout window of up to 16 clock cycles is possible.

\footnotetext{
${ }^{1}$ ToT is measured in units of the clock cycle, which is 25 ns.
} 
The local events are sent out via a VCSEL ${ }^{2}$ optical transmitter through $\sim 80 \mathrm{~m}$ optical fibers to the offdetector readout electronics. Data are received by a PINdiode and then, after conversion to the required format, transferred to the global ATLAS readout system. In the reverse direction another optical connection is used to transmit the LHC clock, bi-phase encoded with commands or configuration data, to the on-detector electronics.

The detector dissipates in total about $10 \mathrm{~kW}$ of power and requires cooling for keeping module temperature at about $-10^{\circ} \mathrm{C}$ to minimize radiation effects in the sensor. The $C_{3} F_{8}$ evaporative cooling, integrated via pipes into the carbon support structure of the detector, is used for this purpose. The cooling system is capable of going down to about $-25^{\circ} \mathrm{C}$ to cope with further radiation-induced damages.

\section{Commissioning}

\subsection{Operation conditions}

The detector was cooled down as a whole for the first time at the end of August 2008. Since then the tuning and the calibration program of the detector has been ongoing. During the single LHC beam injections on September 10th the HV bias of the Pixel sensors was off because of the risk of high charge deposition and therefore the damage of the sensors due to unstable beam. The detector was timed-in with the rest of ATLAS and the first cosmic tracks were recorded on September 14th. During the Fall 2008 cosmic ray data-taking, more than 400 thousand tracks through the Pixel Detector volume were detected, mostly in the barrel region because of the detector geometry. Three cooling circuits in the disks were switched off during data-taking due to a suspected high leak rate of the coolant. They were used however for the calibration, yielding $98.5 \%$ of the detector calibrated in 2008. The measurements of these cooling circuits in 2009 have shown much lower leak rates, which were found acceptable for the operation. However the studies of possible risks of having $C_{3} F_{8}$ coolant in the harsh radiation environment of the LHC based on the leak rate calculations are on-going.

Adjustment of the Pixel module operation temperature is accomplished by the regulation of the so-called backpressure, which is the pressure at the exhaust of the cooling circuits. It was set to 3 bar absolute during 2008 operation, resulting in a spread of the operational temperature from $-3{ }^{\circ} \mathrm{C}$ for most of the barrel modules to $-6{ }^{\circ} \mathrm{C}$ for the disk modules. To achieve nominal operation conditions the temperature of the modules is lowered by about $10^{\circ} \mathrm{C}$ in 2009 using the 2 bar absolute back-pressure.

\subsection{Tuning of optical connections}

The optical connections between the off- and on-detector readout electronics require adjustments in both directions.

\footnotetext{
${ }^{2}$ Vertical Cavity Surface Emitting Laser.
}

The tuning of the "to-detector" connection is required for the timing-in of the individual modules. Due to the propagation delays in the clock distribution circuitry, the LHC clock has different phase at the level of individual module. These individual propagation delays were calculated from the measurements of fiber lengths performed during detector construction and used for the compensation. The tuning of the "from-detector" connection requires adjustment of three parameters: the power of the on-detector VCSEL, the sampling clock phase and the threshold of the PIN diode current in the off-detector receiver. This is accomplished by scanning the parameter space and measuring the bit error rate at each point. Two kinds of bit patterns are used for this purpose: a data pattern, consisting of alternating zeros and ones (so called half-clock pattern) and, only for some special cases, a pseudo-random data pattern, which better mimics the real data transmission. From the bit error rate data an algorithm chooses the optimal operating point [4].

\subsection{Tuning and calibration of FE electronics}

Each pixel cell has an injection circuit that is able to inject a charge into the preamplifier. By repeating the injections and measuring the hit probability as a function of injected charge we obtain a curve, that represents the convolution of the ideal step function and a Gaussian distributed noise. From the curve fit we find the discriminator threshold value as a charge which corresponds to $50 \%$ hit probability and the noise. Using this technique for validation, the thresholds of individual pixels are adjusted to a target threshold. For the 2008 operation the target threshold value of $4000 e^{-}$was chosen. Results of the threshold tuning on the whole detector are shown in Fig. 1(a). As one can see, it is possible to tune the pixels to $4000 e^{-}$ threshold with a very low dispersion of only about $40 e^{-}$. The threshold-to-noise ratio in Fig. 1(b) was measured to be about 24 for most of the pixels and the bump at 13 corresponds to special pixels on the border between FE chips. Further tunings with lower target thresholds are planned before LHC operation restart in 2009.

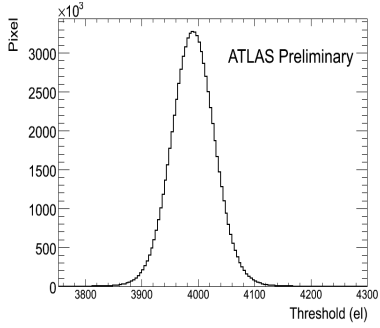

(a)

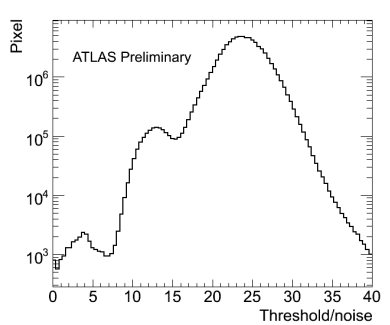

(b)
Figure 1: Results of the threshold measurement per pixel in the detector after the threshold tuning to $4000 e^{-}$(a) and the corresponding threshold-to-noise ratio per pixel (b).

Another tuning is performed by adjusting the preamplifier feedback current, which defines the length of the 
discriminator pulse, or ToT, in response to a certain input reference charge. The reference charge is chosen as 20 $\mathrm{ke}^{-}$, which is the most probable energy loss for a minimum ionizing particle (MIP) in the $250 \mu \mathrm{m}$ Pixel sensor. By injecting this charge into the preamplifier, the individual pixel response is tuned to the ToT of 30 clock cycles. With such a ToT tuning a deposited charge of up to 8 MIPs can be measured. The dependence of the discriminator pulse length on the input charge was measured and incorporated into detector simulation after parameterization.

Several other calibration measurements such as the measurement of the sensor leakage current, the cross-talk between the pixel cells and measurement of the timing characteristics of the FE electronics were also performed.

\subsection{Noise performance}

Even with the discriminator threshold of $4000 e^{-}$and the corresponding high threshold-to-noise ratio there are some pixels with a high noise level. These could be pixels with merged bump bonds, for example. Such pixels would increase data volume sent out to the off-detector readout electronics and therefore limit the maximal readout rate. To spot these pixels the noise occupancy per pixel in the detector is measured using standalone data-taking runs, reading out the detector randomly with the trigger frequency of several $\mathrm{kHz}$. Pixels with an occupancy greater than $10^{-5}$ are defined as noisy and in total about $10^{4}$ (less than $0.02 \%$ of the detector) of such pixels were found.

After masking of these noisy pixels, the noise occupancy in the detector is measured to be about 0.1 hits per event per clock cycle for the whole detector. This corresponds to about $10^{-10}$ noise occupancy per pixel per clock cycle for the whole detector.

\section{Cosmic ray data analysis}

\subsection{Timing-in}

To time-in the detector with the rest of ATLAS, the trigger latency in the Pixel off-detector trigger distribution circuitry was adjusted. For the cosmic ray data-taking a readout window of 8 clock cycles has been used. After fine adjustments of the clock propagation delays in the off-detector Pixel readout electronics, the majority of hits from muon tracks were found within 2 clock cycles. Random phase of the hits with respect to the LHC clock also contributes to this broadening. Therefore we are expecting to use 5 or even 3 clock cycles readout window for initial LHC collisions without losing efficiency.

\subsection{Deposited charge and efficiency}

In Fig. 2 the charge distribution of clusters on tracks, nearly perpendicular to the sensor surface, is shown. It peaks within $5 \%$ to the expected value of $20 \mathrm{ke} e^{-}$and the shape of the curve is in good agreement with expectation from Monte Carlo (MC) simulation. This agreement is also

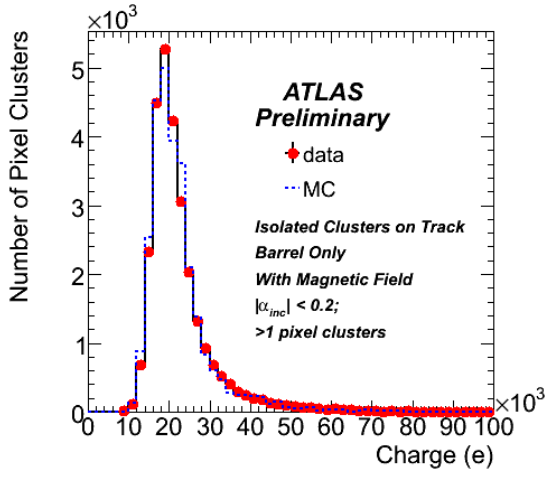

Figure 2: Charge distribution of Pixel clusters from the reconstructed cosmic muon tracks with nearly normal to the sensor surface incidence angle $\left|\alpha_{\text {inc }}\right|<0.2 \mathrm{rad}$, compared to the simulated distribution.

a demonstration of the good quality of the ToT calibration and its parametrization constants used in the simulation.

The efficiency was calculated using the entire ATLAS tracking system [5] by extrapolation of reconstructed tracks to the sensitive volume of the Pixel Detector, considering only operating detector components. The efficiency from muon tracks hits was found to be greater than $99.7 \%$, which is very close to the expected value of $99.9 \%$ from the test beam studies [6].

\subsection{Alignment}

Initially the alignment of the detector is determined from the survey data, collected during detector construction. In-situ only the track-based approach is possible, based on the minimization of the residuals, i.e. the distance between the measured and extrapolated position of the track. The first studies of the cosmic muon hit residuals have shown a significant bow of several barrel staves ${ }^{3}$ with a sagitta of up to $400 \mu \mathrm{m}$. It has been corrected in the detector alignment. Precise measurements thereafter of the distributions of residuals both in the $\mathrm{R} \phi$ plane (referred to as local $x$ - precision coordinate) and in $z$ direction (referred to as local $y$ ) in the Pixel barrel are shown in Fig. 3. The width of each residual distribution, obtained by a Gaussian fit, is compared to the simulation that contains an accurate description of the detector geometry. Apart from the misalignments these widths include contributions from the intrinsic detector resolution and track extrapolation errors. The value for the $z$ - coordinate of $131 \mu \mathrm{m}$ is very close to the simulation value of $127 \mu \mathrm{m}$, whereas for the precision direction some improvement from measured $24 \mu \mathrm{m}$ to the $\mathrm{MC}$ perfect value of 16 $\mu \mathrm{m}$ is still needed. This improvement is expected to be achieved with early collision data, which are also required for the alignment of the endcaps.

\footnotetext{
${ }^{3}$ the building element of the barrel layers comprised of 13 modules sharing the same support structure.
} 


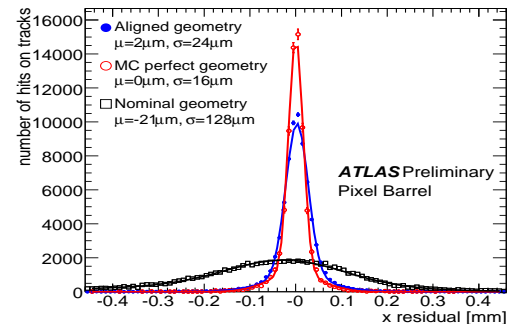

(a)

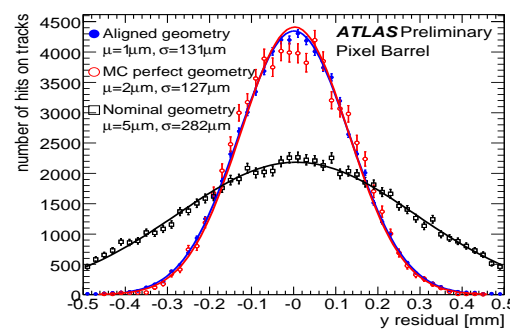

(b)

Figure 3: Distribution of the residuals in local $x$ direction (in $\mathrm{R} \phi$ plane) (a) and local $y$ direction (along $z$ coordinate) (b) for nominal, preliminary aligned and MC simulated geometry. The widths of the distributions include misalignment, detector intrinsic resolution and track extrapolation error components.

\subsection{Lorentz angle measurement}

To measure the momentum of the particles the ATLAS tracking system is operated in a $2 \mathrm{~T}$ solenoidal magnetic field, parallel to the beam axis. The field causes a deflection of the drift of the charge carriers - electrons - in the Pixel sensor from the electric field direction normal to the surface of the sensor. This deflection is characterized by the Lorentz angle and is an important characteristic since it affects the position of the detected charge.

The Lorentz angle is measured from the distribution of cluster charge width as a function of incidence angle of the cosmic muon tracks, which is shown in Fig. 4(a) for both cases with magnetic field on and off. The minimum of the distribution corresponds to the Lorentz angle. For the measurement without magnetic field the Lorentz angle is compatible with zero, as expected. With the magnetic field the measured value of $(213 \pm 0.5) \mathrm{mrad}$ agrees within $5 \%$ with the expected value of $225 \mathrm{mrad}$. Since there is a certain spread of the module temperatures in the detector the Lorentz angle temperature dependence can be studied. The calculations, based on the decrease of the mobility of the charge carriers with the temperature, predict linear dependence with a slope of $d \alpha_{L} / d T=-0.74 \mathrm{mrad} / \mathrm{K}$. The results of the measurement fitted by the straight line are shown in Fig. 4(b). The measured value of $(-0.76 \pm 0.18)$ $\mathrm{mrad} / \mathrm{K}$ agrees well with the calculated one.

\section{Summary}

The complete Pixel Detector has been operated for the first time from August 2008 until the end of the year.

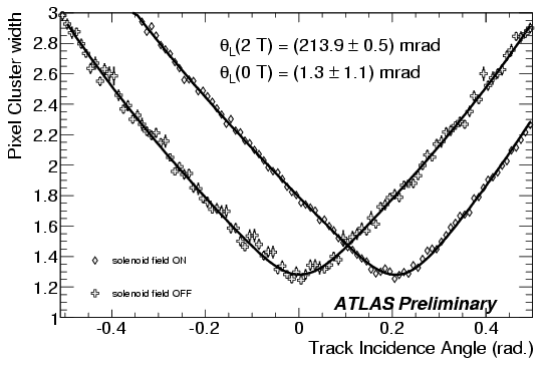

(a)

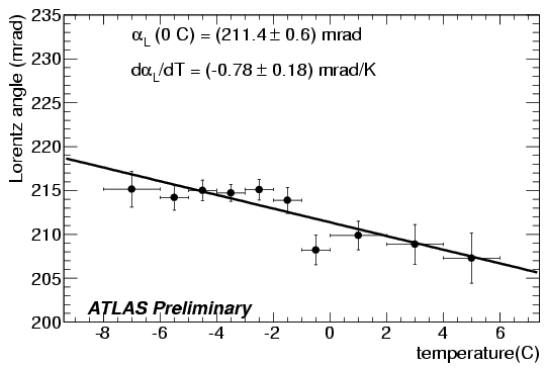

(b)

Figure 4: (a) Pixel cluster size as a function of the track incidence angle for the measurements with and without $2 \mathrm{~T}$ magnetic field. The minimum of both distributions corresponds to the Lorentz angle. (b) Dependence of the measured Lorentz angle on the module temperature.

During this time $98.5 \%$ of the detector channels were extensively tuned and calibrated. After masking of less than $0.02 \%$ channels the detector has shown excellent noise occupancy of $10^{-10}$ and hit efficiency greater than $99.7 \%$. More than 400 thousand cosmic muon tracks were recorded in combined runs with the rest of ATLAS. With this statistics, the detector alignment has been significantly improved in the barrel region. Further improvements in the alignment especially in the endcap region are expected with the first LHC data in November 2009.

\section{Acknowledgment}

This paper is the result of individual contributions of many members of the ATLAS Pixel Detector Group, whom I would like to thank.

\section{References}

[1] The ATLAS Pixel Detector Community, ATLAS Pixel Detector Technical Design Report, CERN-LHCC-98-013.

[2] G. Aad et al., ATLAS pixel detector electronics and sensors, J. Instr. 3 (2008) P07007.

[3] S. Ask et al., The ATLAS Central Level-1 Trigger Logic and TTC System, J. Instrum. 3 (2008) P08002

[4] T. Flick et al., Experiences with the ATLAS pixel detector optolink and researches for future links, 2008 IEEE NSS/MIC Conference Record, Dresden, p. 7-12.

[5] G. Aad et al., The ATLAS Experiment at the CERN Large Hadron Collider, J. Instr. 3 (2008) P08003.

[6] A. Andreazza on behalf of the ATLAS Pixel Collaboration, Nucl. Instr. and Meth. A 565 (2006), p. 23-29. 